

\title{
VIVIENDA SUSTENTABLE: UNA DISCUSIÓN SOBRE EL MANEJO EFICIENTE DEL USO AGUA EN INSTALACIONES DOMICILIARIAS. CASO DE ESTUDIO: SANTA FE -ARGENTINA
}

\author{
SUSTAINABLE HOUSING: A DISCUSSION ON THE EFFICIENT \\ MANAGEMENT OF WATER USE IN DOMESTIC INSTALLATIONS. \\ CASE STUDY: SANTA FE -ARGENTINA
}

\author{
RODOLFO BELLOT \\ Arquitecto \\ Docente e Investigador, Profesor Titular, \\ Facultad de Arquitectura, Diseño y Urbanismo \\ Universidad Nacional del Litoral \\ Santa Fe Argentina \\ https://orcid.org/0000-0003-1929-7820 \\ rjbellot@fadu.unl.edu.ar
}

\author{
DIEGO FISCARELLI \\ Doctor en Arquitectura \\ Docente e investigador, Becario Posdoctoral-CONICET, \\ Facultad de Arquitectura y Urbanismo \\ Universidad Nacional de La Plata \\ La Plata, Argentina \\ https://orcid.org/0000-0002-7087-0816 \\ dfiscarelli@fau.unlp.edu.ar
}

\section{RESUMEN}

Este trabajo busca exponer una reflexión crítica sobre la eficiencia en el manejo del recurso agua, en el marco del caso de estudio -Ciudad de Santa Fe, Santa Fe (Argentina). Ponderando el rol de las instalaciones sanitarias en la optimización del uso del agua como recurso fundamental, se propone, en términos metodológicos, un análisis en base a tres dimensiones: el sistema hídrico de la ciudad, la normativa vigente en materia de regulación de excedentes y el suministro de agua por red. Se recuperan las propias investigaciones precedentes para mensurar el consumo domiciliario típico, atendiendo a dos variables: uso racional del agua y consideración de tecnologías al servicio de la eficiencia. Como síntesis, se elaboran curvas de abatimiento para caracterizar la eficiencia en tres escenarios. Y, en el terreno de la discusión, se interpelan las responsabilidades de los usuarios y la praxis de los profesionales de la arquitectura, encargados de concebir la sustentabilidad de la vivienda urbana santafesina.

\section{Palabras clave}

vivienda, sustentabilidad, eficiencia, agua, curva de abatimiento.

\section{ABSTRACT}

This work aims to present a critical reflection about the efficiency of water resource management in the case study - City of Santa Fe, Santa Fe (Argentina). Analyzing the role of sanitary installations in optimizing water use as an essential resource. In methodological terms, a three-dimensional analysis is proposed: the city's water system, the current regulations for surplus management and the water network supply. The author's prior research projects are recovered to measure typical household consumption, considering two variables: rational water use and consideration of service efficiency technologies.In summary, abatement curves are made to characterize the efficiency in three scenarios. As a discussion, the responsibilities of users, and the praxis of architecture professionals, responsible for conceiving the sustainability of urban housing in Santa Fe, are questioned 


\section{INTRODUCCIÓN}

La arquitectura ha procurado a lo largo de la historia concebir un hábitat eficientemente acondicionado, tanto para seguridad como estancia en el desarrollo de actividades. Sin embargo, fue en el siglo pasado cuando estas preocupaciones alcanzaron una exponencial relevancia. En consecuencia, los pares dialécticos diseño/ tecnología y proyecto/instalaciones consolidaron un entusiasmo científico-tecnológico en pro de la baja de emisiones, el diseño sustentable y el mejoramiento de las condiciones de eficiencia del hábitat

El "nuevo régimen"1, caracterizado por el agotamiento de los recursos energéticos producto de la crisis de los combustibles fósiles en los años 70 -además del cambio climático resultante del alto nivel de emisiones-, obligó a la arquitectura a reflexionar sobre las prácticas proyectuales. Como sostiene Fernández Rojas (cit. en Delucchi 2016, p. 23), se hicieron necesarias "medidas más responsables respecto del abuso y derroche que conlleva una arquitectura de la representación de la espectacularidad, por delante de criterios sencillos y sostenibles de aplicación".

Atendiendo a este panorama, este trabajo propone estudiar el agua como recurso limitado $^{2}$ en la ciudad de Santa Fe (Santa Fe, Argentina). Se plantea como objetivo un análisis que conduce hacia una reflexión sobre el rol de las instalaciones domiciliarias como recurso proyectual, orientado al aprovechamiento del agua $y$, consiguientemente, a la adecuación de la vivienda al medio ambiente.

La discusión involucra entonces a los profesionales, pero también a los usuarios, que con sus hábitos y decisiones afectan desempeño del recurso (Alonso-Frank y Kuchen, 2017). Sibien se reconoce, en diversos autores, antecedentes que describen similares trayectos de investigación, este trabajo pretende aportar una perspectiva innovadora al estudiar el caso de la ciudad de Santa Fe, en el marco de la reciente normativa de regulación hídrica. En este sentido, la contribución fundamental radica aquí en la revisión crítica del caso instrumental en relación con el alcance del marco regulatorio, aun cuando se recupera los caracteres metodológicos de análisis previos.

En términos de características arquitectónicas, debe indicarse que a pesar de que este artículo recorta en la utilización de dispositivos regulares pluviales como variable central -dada la normativa vigente-, a fin de efectuar un estudio comparativo, los criterios de diseño y resolución constructiva de las edificaciones inciden directamente en la eficiencia hídrica. A partir de dicho estudio, se constata que la planificación del tamaño y pendiente de las cubiertas, al igual que la manipulación de la capacidad absorbente en los casos que incluyen superficies verdes, consolidan pautas para optimizar la reutilización del agua, en oposición a aquellos casos que sólo proponen su evacuación.

\section{METODOLOGÍA}

Desde el punto de vista metodológico, para arribar a la discusión final, este trabajo plantea dos fases, que se detallan a continuación:

Fase 1: Análisis multidimensional del caso de estudio: Ciudad de Santa Fe.

Dimensión 1: Se caracteriza la cuenca hidrográfica que compromete a la ciudad, con el objeto de ponderar la interrelación entre las características geográficas del área y los particulares regímenes pluviométricos e hídricos. Asimismo, se describe el origen de los dispositivos reguladores pluviales en el marco de diversas decisiones, que han pretendido dar respuesta las especificidades de la región en términos de sus condiciones naturales.

Dimensión 2: Se caracteriza la normativa vigente en materia de regulación de excedentes pluviales.

Dimensión 3: Se cuantifica en profundidad el suministro de agua por red, recuperando datos de investigaciones precedentes LATEC/FAU/LATMAT/FADU y cátedras de Instalaciones FADU-UNL.

Fase 2: Análisis del caso de estudio (3 dimensiones), a partir de los tres escenarios siguientes:

1. Nivel de eficiencia del usuario y cambio en los hábitos de consumo (Alonso-Frank y Kuchen, 2017)

2. Cambio en la tecnología sin uso del agua de lluvia cosechada

3. Cambio en la tecnología con uso de agua de lluvia cosechada

1 "Nuevo régimen climático" es un término acuñado por Bruno Latour. En su último libro (2017), utiliza este concepto haciendo alusión al fin del Holoceno y comienzo del Antropoceno.

2 Según la empresa Aguas Santafesinas S.A., la cantidad de energía necesaria -bombeo, transporte, tratamiento y distribución- para obtener 1 metro cúbico de agua apta para el consumo humano desde la fuente de río, oscila entre 0.35 Y $0.40 \mathrm{Kw} / \mathrm{h} / \mathrm{m} 3$. De esta manera, aunque este trabajo reconoce la interrelación entre los recursos hídrico y energético en el contexto de la provisión domiciliaria, tal aspecto es intencionalmente omitido en el análisis de la eficiencia en el manejo del agua como recurso fundamental. 


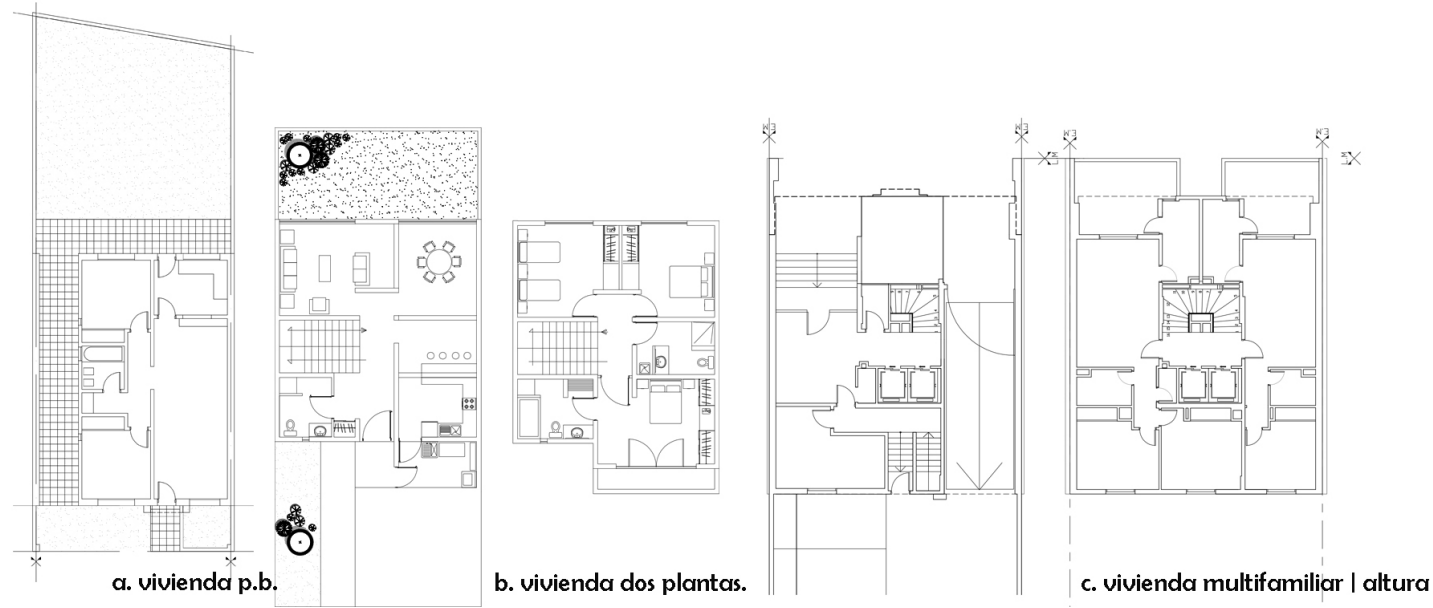

Figura 1. Tipologías para el análisis. Fuente: Elaboración de los autores.

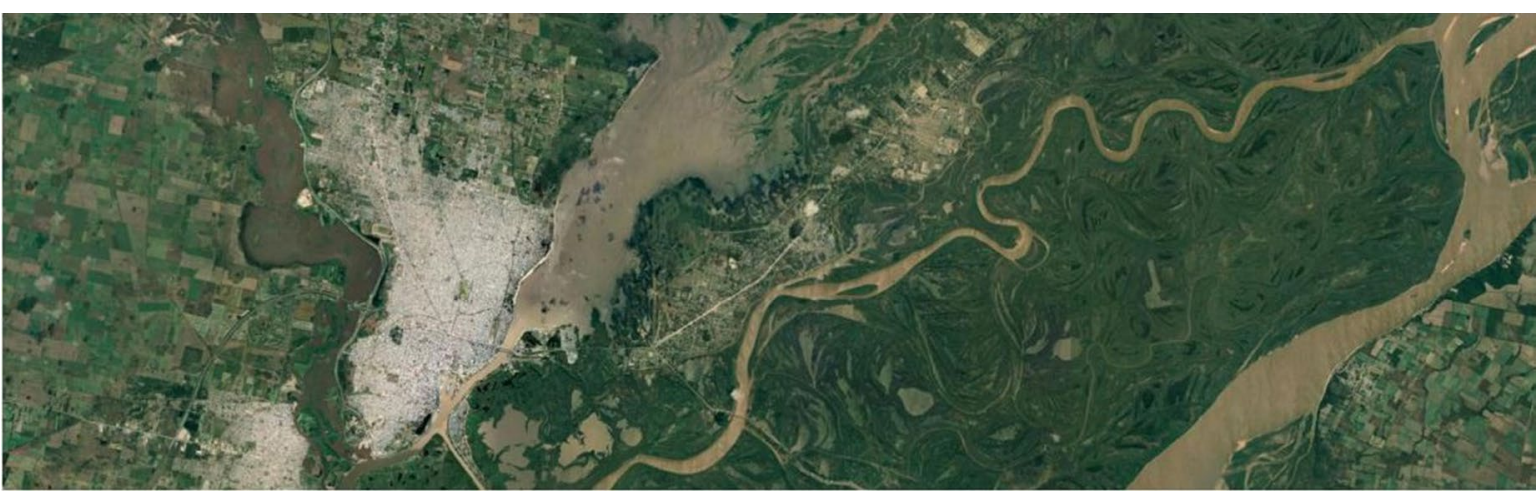

Figura 2. Foto aérea metropolitana del Gran Santa Fe. Fuente: Elaboración de los autores en base a Google Earth.

En el desarrollo de esta segunda fase del análisis, se contemplan tres tipologías posibles de viviendas con instalación sanitaria tradicional, para servir un promedio de cuatro integrantes ${ }^{3}$, las que cuentan con: un baño, una cocina, un lavadero y una canilla de servicio para un patio pequeño o mediano (Figura 1). Las tipologías son:

a. Vivienda de una planta o planta baja

b. Vivienda de dos plantas o pisos altos

c. Vivienda en edificio multifamiliar o en altura.

Y para elaborar una síntesis de cada escenario de eficiencia, se retoma el concepto de "abatimiento" (Clerc, Díaz y Campos, 2013), mediante el cual se entienden los costos como beneficios percibidos al reemplazar una tecnología o uso de referencia por una alternativa. De este modo, se puede elaborar una curva, en cuanto representación gráfica de los costos abatidos de un menú de opciones, junto a sus porcentajes de reducción.

\section{ANÁLISIS}

\section{DIMENSIÓN 1: EL SISTEMA HÍDRICO DE SANTA FE}

La ciudad de Santa Fe está asentada en la confluencia y valle de inundación de dos ríos: Paraná y Salado. Sus límites físicos son, en su mayoría, fluviales, y además el $70 \%$ de su territorio está conformado por humedales (lagunas, ríos y bañados).

De acuerdo con Sánchez y Sánchez (2004), el aumento sostenido en la demanda sobre los recursos hídricos es limitado, poniendo de manifiesto la necesidad de buscar formas más eficientes para aprovecharlos, como también de considerar medidas que moderen su uso en procesos $o$ actividades.

Asimismo, Santa Fe se ubica en la cuenca hidrográfica del Río Paraná, área que presenta como característica constate 
eventos hidroclimáticos cíclicos, con abundantes Iluvias, crecidas y desborde de ríos, principalmente en los periodos de verano y otoño. La ubicación en esta cuenca, denominada técnicamente "Paraná Medio" recibe una influencia de todos los fenómenos que suceden en la parte alta, Bolivia, Brasil y Paraguay (Figura 2).

El Río Paraná define el límite hacia el Este, mientras que el Río Salado, el del Oeste, y pese a las particularidades, sus valles de inundación llegan a tener contacto en el borde sur de la ciudad, situación que magnifica la vulnerabilidad del territorio. Así, y retomando a Paoli y Schreider (2000), el Río Paraná ${ }^{4}$ es, sin duda, el más importante del sistema hidrográfico "Río de la Plata", al contar con una superficie de cuenca de $1.510 .000 \mathrm{~km}^{2}$ y una extensión de, aproximadamente, unos $2.570 \mathrm{~km}$.

Las características geográficas de la ciudad han conllevado consecuencias históricas. Desde 1905 se han registrado numerosos períodos de creciente e inundaciones ${ }^{5}$, pero fue en el año 2003 y 2007 cuando la ciudad atravesó dos fenómenos significativos que ameritaron la formulación de un Plan Director. ${ }^{6}$ En él, se pautaron acciones estructurales: consolidación de defensas, construcción de nuevos puntos de operación para la extracción de agua de la ciudad, conformación de reservorios para la acumulación de agua de lluvia y un complejo sistema de drenajes. En paralelo, se implementaron disposiciones legales para el tratamiento de residuos que afectan los desagües, la creación de cintas verdes ${ }^{7}$, un nuevo factor de impermeabilización del suelo (FIS), modificaciones al reglamento de ordenamiento urbano -ROU- y sistemas de regulación de excedentes pluviales.

Resulta además necesario caracterizar, por un lado, el régimen hídrico de los ríos, y por el otro las precipitaciones.

En relación con lo primero, todo el sistema Paraná Medio cuenta con crecidas producidas por los aportes en las zonas altas: "[...] la génesis de sus aportes indica que

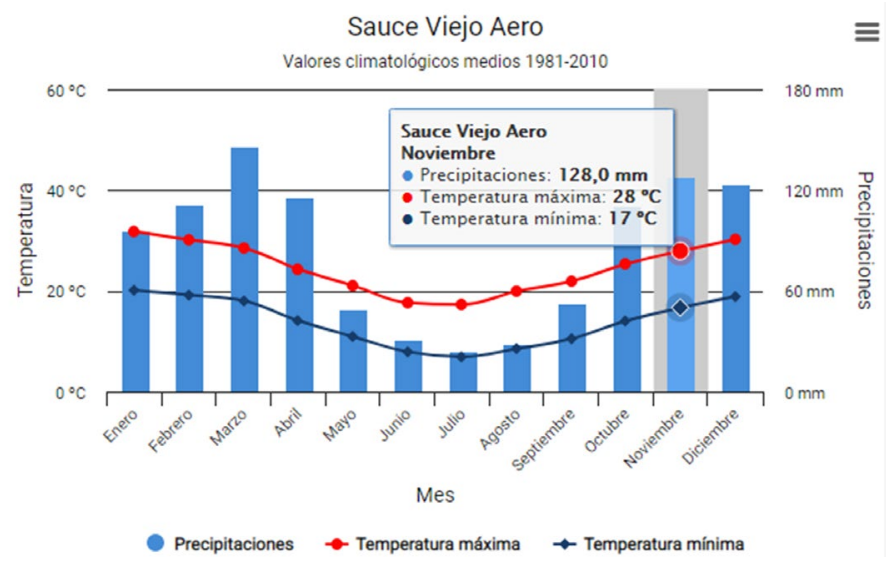

Figura 3. Precipitaciones mensuales Santa Fe, estación Sauce Viejo. Fuente: Servicio Meteorológico Nacional, 2019.

las lluvias que se producen en las cuencas de aporte del Alto Paraná, Río Iguazú y Río Paraguay, son las causantes directas de los derrames en los tramos medio e inferior, presentando una baja incidencia los aportes propios de dichos tramos" (Paoli y Schenider, 2000, p. 72). De acuerdo con los autores, el Río Paraná -a la altura de la provincia de Corrientes, Argentina- tiene un módulo anual de $17000 \mathrm{~m}^{3} / \mathrm{s}$, recibiendo aportes del Paraná Alto y el Paraguay, y en todo el tramo medio, incorpora unos $1000 \mathrm{~m}^{3} / \mathrm{s}$.

Respecto del régimen de precipitaciones, debido a la escasez pluvial en invierno y los excesos en verano, se presenta con un promedio anual de entre $1000 \mathrm{~mm}$ a 1200 mm (Figura 3). Acorde con el Servicio Meteorológico Nacional (SMN), las precipitaciones no distan de las mencionadas para toda la Cuenca: con 1019,80 mm anuales y máximas extremas de hasta casi $200 \mathrm{~mm}$ diarios registrados en el mes de marzo de 2007.

4 La cuenca del Paraná incluye territorio de Brasil, Argentina, Paraguay y Bolivia, abarcando una superficie de 2.6 millones de kilómetros² (84\% del sistema fluvial del Río de la Plata), e integra el zócalo brasileño y las vertientes orientales de un sector andino a través de su recorrido por Brasil, Bolivia, Paraguay y Argentina. El sistema está compuesto por tres áreas hidrográficas: el Río Paraguay, el Alto Paraná y el Paraná Medio e Inferior (Bello, Ballesteros, Buitrago, González y Velasco, 2018).

5 Inundaciones por crecidas del Río Paraná: 1905, 1966, 1982/3, 1992, 1998; por crecidas del Río Saldado: 1914, 1973, 2003; por lluvias extraordinarias; 2007 (Informe 2019 de la Gestión de Riesgos de Santa Fe).

6 Plan Director de desagües pluviales urbanos, formulado en el marco del Plan Urbano 2010. Gobierno de la Ciudad de Santa Fe.

7 Cintas verdes refiere a un espacio con césped y/o árboles que se debe contemplar al momento de construir una vereda y destinado a una mejor absorción del agua de lluvia, disminuyendo la superficie construida con solados. En la ciudad de Santa Fe es obligatoria por ordenanza y en todos los casos en los cuales el ancho de la vereda o acera supere los dos metros. Véase: Ordenanza N¹1610. Honorable Concejo Municipal de la Ciudad de Santa Fe de la Vera Cruz. 17/9/2009. 

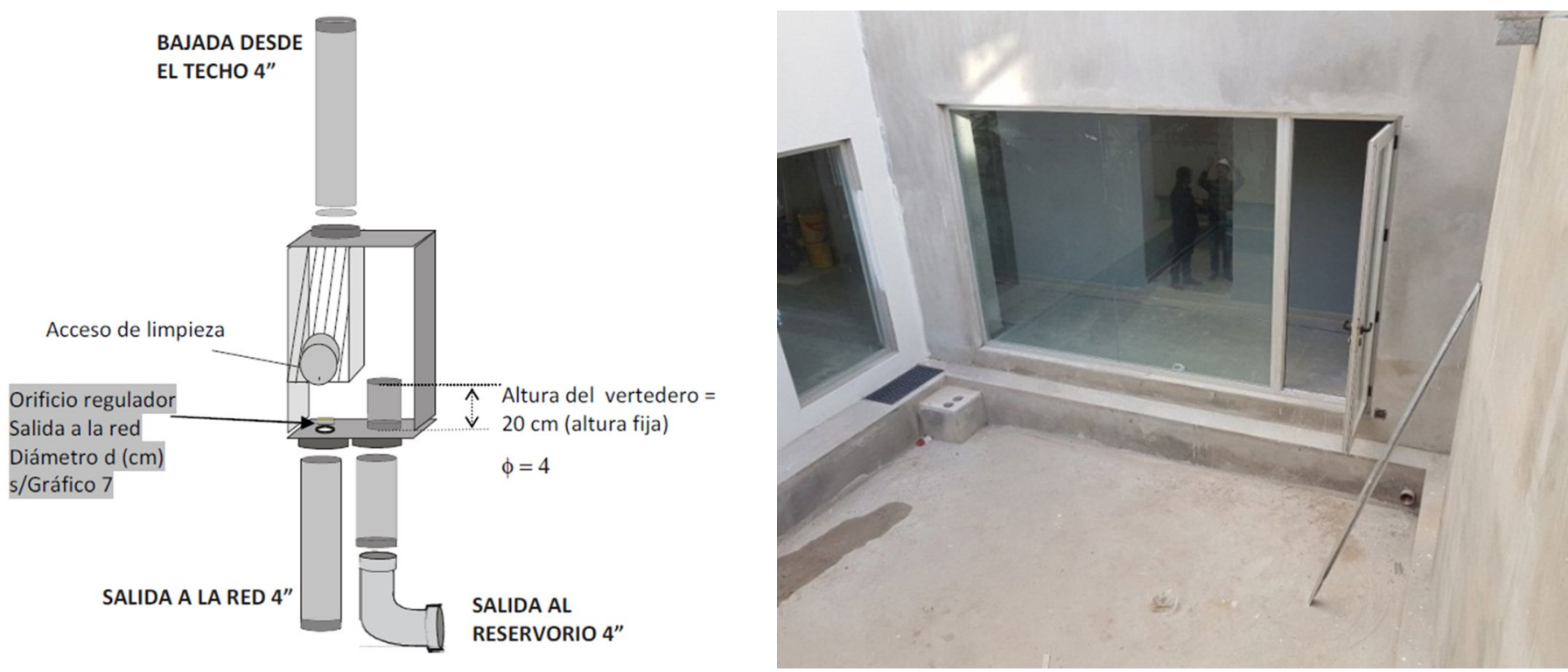

Figura 4. Dispositivo regulador hidráulico. Fuente: Izquierda: Secretaría de Recursos Hídricos de Santa Fe.

Figura 5. Reservorio diseñado en el edif. SJ 58 Pilay-Capitel - calle Irigoyen Freyre 2230, Sta. Fe.

El patio de PB es en sí mismo el reservorio, ocupando éste toda su superficie. Fuente: Fotografía de los autores.

\section{DIMENSIÓN 2: NORMATIVA Y REGULACIÓN DEL EXCEDENTE}

En diciembre de 2012 se sancionó en la ciudad la Ordenanza N 11959 "Sistema de regulación de excedentes pluviales" 8. El modo con el que se propone contribuir con la optimización del funcionamiento del sistema urbano consiste en la incorporación de dispositivos hidráulicos que tendrían por misión controlar la evacuación de excedentes pluviales de una forma paulatina, retardando los drenajes para controlar su impacto en el sistema. Estos dispositivos hidráulicos deberán reducir el caudal máximo a un mínimo del $50 \%$. Y se componen de dos elementos conceptuales: un "reservorio" y un "regulador".

En cuanto a las instalaciones domiciliarias, el sistema funciona del siguiente modo: el agua escurre por cubiertas y superficies impermeables hacia canaletas y embudos; luego es transportada hacia tuberías de bajada en las que se interpone un "regulador", que conduce, a través de un ducto con orificio de salida reducido, el agua de lluvia a la red, y el resto -rebalse-, mediante uno estándar que se redirige y almacena en un "reservorio". En éste, y de igual manera, una parte es escurrida a la red por medio de una salida con orificio regulado, y el resto se almacena en la cisterna. Para determinar las capacidades y diámetros de los componentes, se realiza un simple cálculo que consiste en determinar las superficies impermeables del edificio y considerar las pendientes de cubiertas. Con estos datos, se considera una curva de retardo del 50\%; luego, a partir de la elaboración de tablas, se obtiene el volumen del reservorio y se define el diámetro del orificio de salida (Figuras 4 y 5).

\section{EL REGULADOR HIDRÁULICO COMO INSTRUMENTO DE LA NORMATIVA}

A efectos establecer precisiones en el marco del análisis de la dimensión normativa, se propone un ejemplo práctico: una vivienda con una cubierta de techos de $100 \mathrm{~m}^{2}$ y una tormenta de diseño con una intensidad ${ }^{10}$ de $180 \mathrm{~mm} / \mathrm{h}$, lo que significa 18.000 litros de agua que deben ser evacuados.

Considerando la ecuación de Díaz Dorado (2008), el caudal a evacuar para el ejemplo citado es de: $5 \mathrm{l} / \mathrm{s}$.

$\mathrm{Q}($ caudal $)=(\mathrm{S}$ (sup.) $\times$ Imáx (lluvia) $\times$ e (coef. fricción))/3600

El gráfico siguiente muestra las curvas de precipitaciones de la zona analizada en función de la recurrencia, en donde,

8 Ordenanza Nª 11959, Sistema de regulación de excedentes pluviales de Santa Fe de la Vera Cruz. Consejo Municipal, 13 de diciembre de 2012.

9 La ordenanza exige considerar todas las superficies impermeables, solados, etc. Sólo con fines ilustrativos se ofrece como ejemplo una cubierta de techos.

10 "Intensidad de lluvia" es la razón de incremento de la altura que alcanza la lluvia con respecto al tiempo; su unidad de medida es $\mathrm{mm} / \mathrm{h}$ 
con una doble entrada, se indica en la abscisa la duración y en la ordenada la intensidad. De ello, se determina el dato de $180 \mathrm{~mm} / \mathrm{h}$ para los cálculos ulteriores (Figura 6).

Además de considerar el caudal a evacuar por un inmueble, es necesario precisar otro parámetro: el régimen de lluvia. Entonces, se podrá cuantificar la cantidad de agua que se almacena en una cisterna, cumpliendo la normativa No 11959 a lo largo de un período determinado. Se consideran algunos datos para transformarlos en fuentes de información, según el SMN, como se mencionó, el régimen de precipitaciones de la ciudad de Santa $\mathrm{Fe}$, es de entre 1000 a $1200 \mathrm{~mm}$ anuales (Figura 7).

EL SMN posee datos rigurosos en dos periodos (1961/90 y 1981/10), en los cuales se puede advertir que los $1000 \mathrm{~mm}$ a $1200 \mathrm{~mm}$ anuales no se suceden de modo equitativo, siendo los meses de verano más lluviosos los correspondientes al invierno. También se informa la cantidad de días en cada mes y cada estación con días de lluvia. Considerando este dato, por el cual no es factible acumular agua de lluvia de igual forma en todos los meses y estaciones, se propone, con fines prácticos, valorar el régimen de 1000/1200 mm anuales en un promedio lineal.

Como resultado, una vivienda con una cubierta de $100 \mathrm{~m}^{2}$ que recibe $1000 \mathrm{~mm}$ a $1200 \mathrm{~mm}$ de agua anual, recogerá de 120.000 a 144.000 litros por año. De ello, se calcula un promedio mensual de 10.000 a 12.000 litros.

Resta exponer que, si bien la ordenanza No 11959 contempla el almacenamiento para atenuar el impacto hídrico en el sistema urbano, no estipula la cosecha para uso. Entonces, los volúmenes de cisternas, en principio, no cuentan con capacidad plena para acumular los montos de lluvia, por lo que dependerá de las estaciones, los meses y los días con lluvia.

\section{DIMENSIÓN 3: SUMNISTRO DEL AGUA Y DISTRIBUCIÓN POR RED}

De acuerdo al informe "Santa Fe, cómo vamos 2016, 2017 y 2019", producido por el gobierno de la ciudad y la Bolsa de Comercio, al igual que los datos oficiales de la firma Aguas Santafesinas SA (ASSA), más del $92 \%$ de la ciudad cuenta con red de agua potable proveniente de una toma superficial en el Río Colastiné (perteneciente a la cuenca del Río Paraná). El resto de la población se abastece de agua a través de perforaciones comunitarias y domiciliarias. El consumo diario de agua por habitante en la localidad es de 397,5 litros, siendo el recomendado por la OMS de 100 litros per cápita (Bartram et al., 2009), vale decir que se cuadriplican los valores estipulados. Este indicador promedio de consumo surge, según ASSA, de suponer el producido distribuido entre los usuarios. En concordancia con lo que afirma Franco (2019), el consumo real debe operarse con sistemas de medición y no por distribución de producción entre los servidos. Como

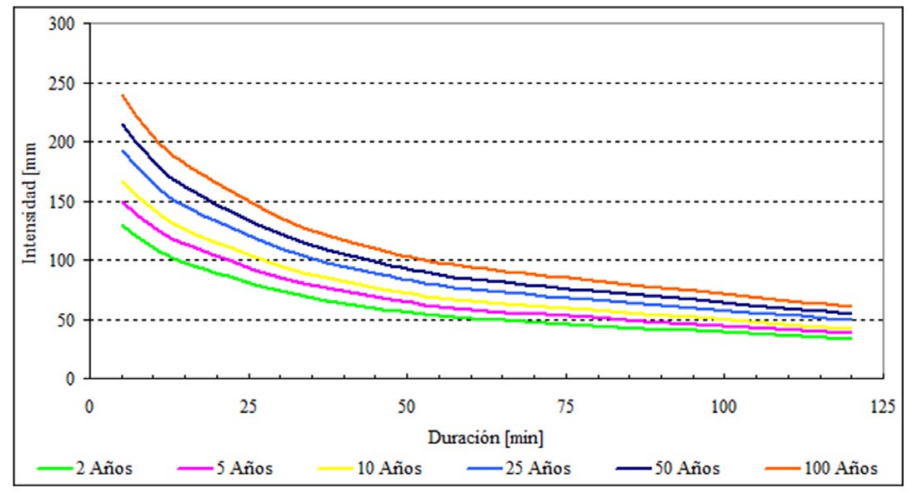

Figura 6. Curvas de intensidad-duración-frecuencia IDF serie 1970 2006 (Bertoni) II - Taller de Regionalización. Fuente: FICH-UNL Facultad de Ingeniería y Ciencias Hídricas de la Universidad Nacional Litoral.
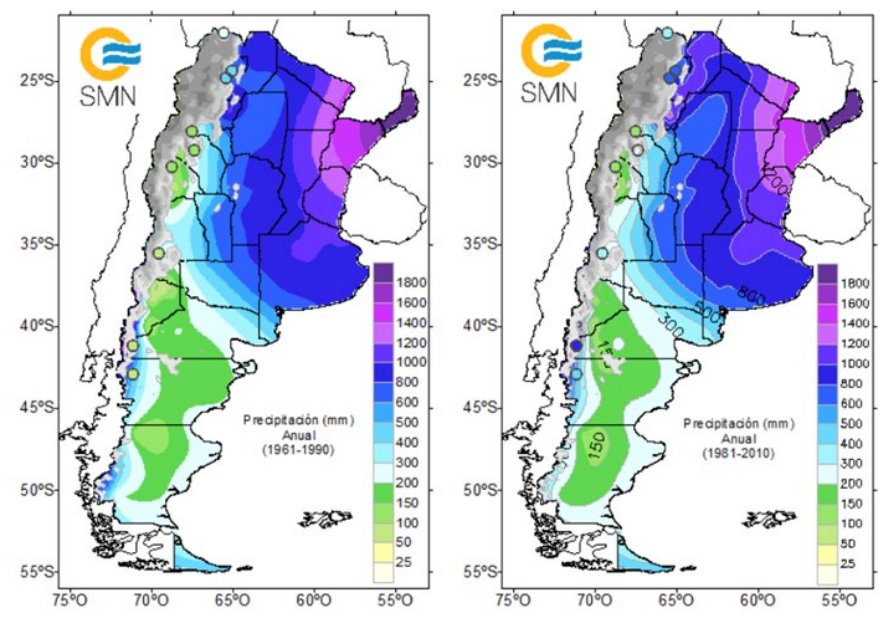

Figura 7. Precipitaciones anuales periodos de (1961-1990) y (1981-2010). Fuente: SMN.

advierten Calcagno, Mendiburo y Gaviño (2000), el promedio de litros/habitantes/día tiene en la Argentina un rango muy amplio de variación entre provincias, que oscila desde un máximo de $6541 / \mathrm{h} / \mathrm{d}$ a mínimos de 1681/h/d.

Los valores de agua no contabilizada constituyen uno de los principales problemas de eficiencia en la mayoría de los servicios de agua potable. Se estima que entre un 35 a un $40 \%$ del agua producida se pierde en las redes y en las conexiones clandestinas. En este sentido, Jiménez (1994) propone una clasificación para el uso eficiente del agua basada en cinco grupos; detección y reparación de fugas, tarifas, educación de usuarios y reglamentaciones.

Según datos de la prestataria ASSA (2019), se encuentran instalados 17.380 medidores, que representan el 16\% del total de conexiones. Esto significa que la mayor parte de los usuarios pagan de acuerdo al sistema tarifario catastral -por asignación de volumen. Por ello, el régimen tarifario en la ciudad, en su mayor porción, está basado en el sistema de volumen de agua asignado mediante catastro y está regulado por la Ley Provincial No 11220 "Régimen Tarifario de la 
Prestación del Servicio"11. Los usuarios conectados al servicio poseen una fórmula de cálculo que se expresa así:

$\mathrm{MF}=(\mathrm{CF}+\mathrm{P} \times \mathrm{Q}) \times \mathrm{FS} \times \mathrm{TR}$

MF Monto a facturar (\$/periodo)

CF Cargo fijo (\$/periodo)

P Precio del $\mathrm{m}^{3}$ de agua $\left(\$ / \mathrm{m}^{3}\right)$

Q Volumen de agua a facturar $\left(\mathrm{m}^{3} /\right.$ periodo - QM medido o QA asignado)

FS Factor de servicio

TR Tasa retributiva control y regulación (ENRESS)

La revisión tarifaria mantiene el esquema de subsidios a los usuarios por parte del Estado Provincial, que seguirá cubriendo con fondos del tesoro parte de los gastos que debe afrontar la empresa para prestar sus servicios. El subsidio estatal en Santa Fe es del orden del 30\% (ASSA, 2019). En virtud de un estudio realizado por la FADU-UNL, en conexiones con medidor instalado por la empresa ASSA, el promedio de agua consumida QM es de 188//h/d, valor que dista de los $397,51 / \mathrm{h} / \mathrm{d}$ declarados por la empresa.

Dado lo expuesto, es posible afirmar que los valores confirman, por un lado, la sobreasignación de consumo para los sistemas no medidos y la diferencia positiva entre el consumo real por persona y el declarado por la prestataria en una brecha de entre el $40 \%$ y $50 \%$.

\section{EFICIENCIA EN EL USO DEL RECURSO}

La normativa de Obras Sanitarias de la Nación (OSN) fijó valores de capacidad de tanques de reserva en función de promedios de consumo diario asignados a unidades locativas tipo que incluían: un baño principal, un baño de servicio, una pileta de cocina y una pileta de lavar (Lemme, 1973). La unidad tipo se consideraba, por cierto, conformada por una familia de cuatro integrantes. El Reglamento para Instalaciones Sanitarias Domiciliarias ${ }^{12}$ establecía que para la unidad tipo se debía contar con un tanque de 850 litros, cuando éste fuera alimentado en forma directa -sin tanque de bombeo-. Por su parte, el Título II (provisión domiciliaria de agua), Capítulo III, art. 142, inc. 1, indica: "[...] deberán tener dimensiones proporcionales a la cantidad de agua que deban proveer y su capacidad útil será, por lo menos, igual al consumo que corresponda a veinticuatro horas de uso". De aquí surge un consumo promedio estipulado por persona y por día de 212,5 litros de agua (Tabla 1 y Figura 8).

\begin{tabular}{|c|c|c|c|}
\hline Higiene personal & Consumo & Uso & $\begin{array}{c}\text { Litros / } \\
\text { Habitante / } \\
\text { Día }\end{array}$ \\
\hline Ducha & $8 \mathrm{~min}$ & 1 uso diario & $75 \mathrm{~L}$ \\
\hline Bañera & $\begin{array}{l}1,70 \mathrm{~m} x \\
0,75 \mathrm{~m}\end{array}$ & 1 uso diario & $200 \mathrm{~L}$ \\
\hline $\begin{array}{l}\text { Descarga de inodoro } \\
\text { selectivo }\end{array}$ & 3 a $6 L$ & 10 descargas & $45 \mathrm{~L}$ \\
\hline Descarga de inodoro & 6 a $10 \mathrm{~L}$ & 10 descargas & $80 \mathrm{~L}$ \\
\hline Válvulas de inodoros & $1 \mathrm{~L} / \mathrm{s}$ & 10 desc. de $3 \mathrm{~s}$ & $30 \mathrm{~L}$ \\
\hline Bidet & $10 \mathrm{~L}$ & 1 minutos & $10 \mathrm{~L}$ \\
\hline $\begin{array}{c}\text { Pileta de lavar las } \\
\text { manos }\end{array}$ & $0,16 \mathrm{~L} / \mathrm{s}$ & 3 minutos & $30 \mathrm{~L}$ \\
\hline Promedio & & & $160 \mathrm{~L}$ \\
\hline \multicolumn{4}{|l|}{ Higiene del hogar } \\
\hline $\begin{array}{c}\text { Lavado de platos y } \\
\text { enseres }\end{array}$ & $0,16 \mathrm{~L} / \mathrm{s}$ & 8 minutos & $18,75 \mathrm{~L}$ \\
\hline Lavadora automática & $4 \mathrm{Kg}$. & 1 usos diarios & $6,25 \mathrm{~L}$ \\
\hline $\begin{array}{c}\text { Pileta de lavar la } \\
\text { ropa }\end{array}$ & $0,16 \mathrm{~L} / \mathrm{s}$ & 5 minutos & $12,5 \mathrm{~L}$ \\
\hline Lavarropas & $\begin{array}{l}50 \text { a } 60 \mathrm{Lp} / \\
\text { ciclo }\end{array}$ & 1 uso & $13,75 \mathrm{~L}$ \\
\hline $\begin{array}{l}\text { Limpieza del hogar } \\
\text { (ej. pisos) }\end{array}$ & $0,16 \mathrm{~L} / \mathrm{s}$ & Promedio & $12,5 \mathrm{~L}$ \\
\hline Lavado de vehículos & $500 \mathrm{~L}$ & $\begin{array}{c}1 \text { uso } \\
\text { semanal }\end{array}$ & * \\
\hline Promedio & & & $45 \mathrm{~L}$ \\
\hline \multicolumn{4}{|l|}{$\begin{array}{l}\text { Mantenimiento de } \\
\text { verde }\end{array}$} \\
\hline Regado de plantas & $0,16 \mathrm{~L} / \mathrm{s}$ & Diario & $4 \mathrm{~L}$ \\
\hline $\begin{array}{l}\text { Regado de jardín } \\
\text { pequeño }\end{array}$ & $0,16 \mathrm{~L} / \mathrm{s}$ & Promedio & $18,75 \mathrm{~L}$ \\
\hline $\begin{array}{l}\text { Regado de jardín } \\
\text { mediano }\end{array}$ & $0,16 \mathrm{~L} / \mathrm{s}$ & Promedio & $37,5 \mathrm{~L}$ \\
\hline Promedio & & & $18 \mathrm{~L}$ \\
\hline \multicolumn{4}{|l|}{ Consumo e ingesta } \\
\hline Para beber & & Diario & $1,5 \mathrm{~L}$ \\
\hline Para cocinar & & Diario & $1,5 \mathrm{~L}$ \\
\hline Promedio & & & $3 L$ \\
\hline Total diario & & & $226 \mathrm{~L}$ \\
\hline
\end{tabular}

Tabla 1. Consumo de agua en función de usos en viviendas considerados en litros/habitante/día. Fuente: Cátedra Instalaciones FADU-UNL (2018).

11 Gobierno de Santa Fe. Ley Provincial N 11220, Transformaciones del sector público de agua potable, desagües cloacales y saneamiento. 12 de diciembre de 1994

12 Decreto N 11877 del P.E.N. - Ministerio de Obras Públicas de la Nación - Administración General de Obras Sanitarias de la Nación - Argentina - 1954 y modificatorias

13 Investigación CAI+D, (2008) denominada "Arquitectura sustentable, desarrollo experimental de un módulo habitacional con consumo de energía 0", bajo la dirección del Prof. Arq. Alberto Maidana, FADU-UNL. 
En ese contexto, la presente investigación recoge el método de identificación de usos de agua domiciliaria propuesto por Castillo-Ávalos y Rovira-Pinto (2013), en tanto formula un cálculo de modo comparativo entre los volúmenes potenciales de agua que se pueden llegar a ahorrar en la vivienda, para lo cual se procedió, por parte del Laboratorio de Técnicas y Materiales (LATMAT) FADU-UNL ${ }^{13}$, a readaptar el método y recalcular los valores de consumo de agua en viviendas pertenecientes a la ciudad de Santa Fe:

\section{ANÁLISIS DESDE 3 ESCENARIOS}

A continuación, recuperando las tres dimensiones sobre las que se construyó el análisis en primera fase y caracterizado el caso de estudio en términos de provisión, aprovechamiento y manejo del agua como recurso, se definen tres escenarios que constituyen situaciones típicas a comparar, con el objetivo de completar el análisis, obtener los resultados y promover la discusión final.

\section{$1^{\circ}$ ESCENARIO - NIVEL DE EFICIENCIA DEL USUARIO}

La primera opción, "nivel de eficiencia del usuario" (NEU), para adoptar el término de Alonso-Frank y Kuchen (2017), se puede incorporar con acciones como las que siguen: Una ducha de $10 \mathrm{~min}$, consume 100 litros de agua, mientras que una de $5 \mathrm{~min}$, la mitad. Si se utiliza un vaso para cepillarse los dientes, se consume 0,25 litros de agua, mientras que dejando abierta la canilla se emplea entre 20 y 30 litros. Afeitarse cerrando el grifo consume unos 3 litros, mientras que hacerlo con el grifo abierto, consume unos 40 litros de agua. Mantener el verde de patios y jardines regando por la tarde/noche o al amanecer en vez de hacerlo mediante gatillos reguladores, permite ahorrar hasta la mitad de agua. Todos estos datos, extraídos de Agua y Saneamientos Argentinos (2019) (AySA) e investigaciones LATMAT, FADUUNL, evidencian que es posible una reducción de hasta el $30 \%$, tan solo a partir de un uso racional y cambios de hábitos en los usuarios-habitantes.

Si se considera el ítem higiene personal (Figura 9), responsable del $70 \%$ del consumo de agua por habitante y por día, se puede graficar el posible uso eficiente en litros para los distintos artefactos involucrados; de lo cual se advierte que el consumo del recurso podría alcanzar una reducción de hasta el $30 \%$, sin alterar niveles de confort. En el ítem higiene del hogar (Figura 10), el NEU puede llegar hasta valores superiores al $20 \%$, por ejemplo, mediante la utilización de baldes para la limpieza de pisos en lugar de mangueras a canilla abierta. En relación al mantenimiento de verde (Figura 11), considerando patios chicos y medianos, el uso eficiente no es tan significativo como en los anteriores, pues llega a valores de hasta 10\% de NEU, gracias al uso de gatillos dosificadores o regaderas. En cuanto al consumo de agua para ingesta (Figura 12), para cocción, bebida e infusiones, no se contempla ningún valor de NEU.

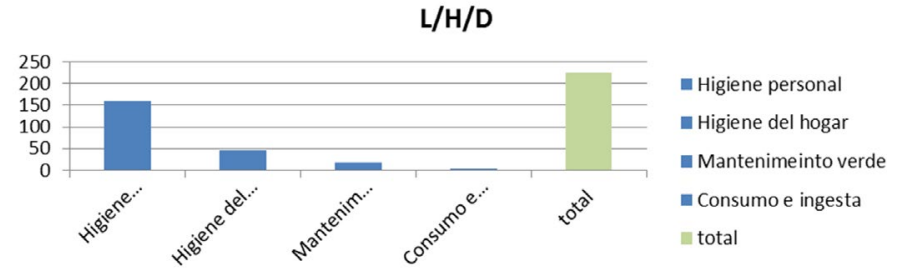

Figura 8. Usos en relación a los consumos de agua. Fuente: Cátedra de Instalaciones FADU -UNL (2018).
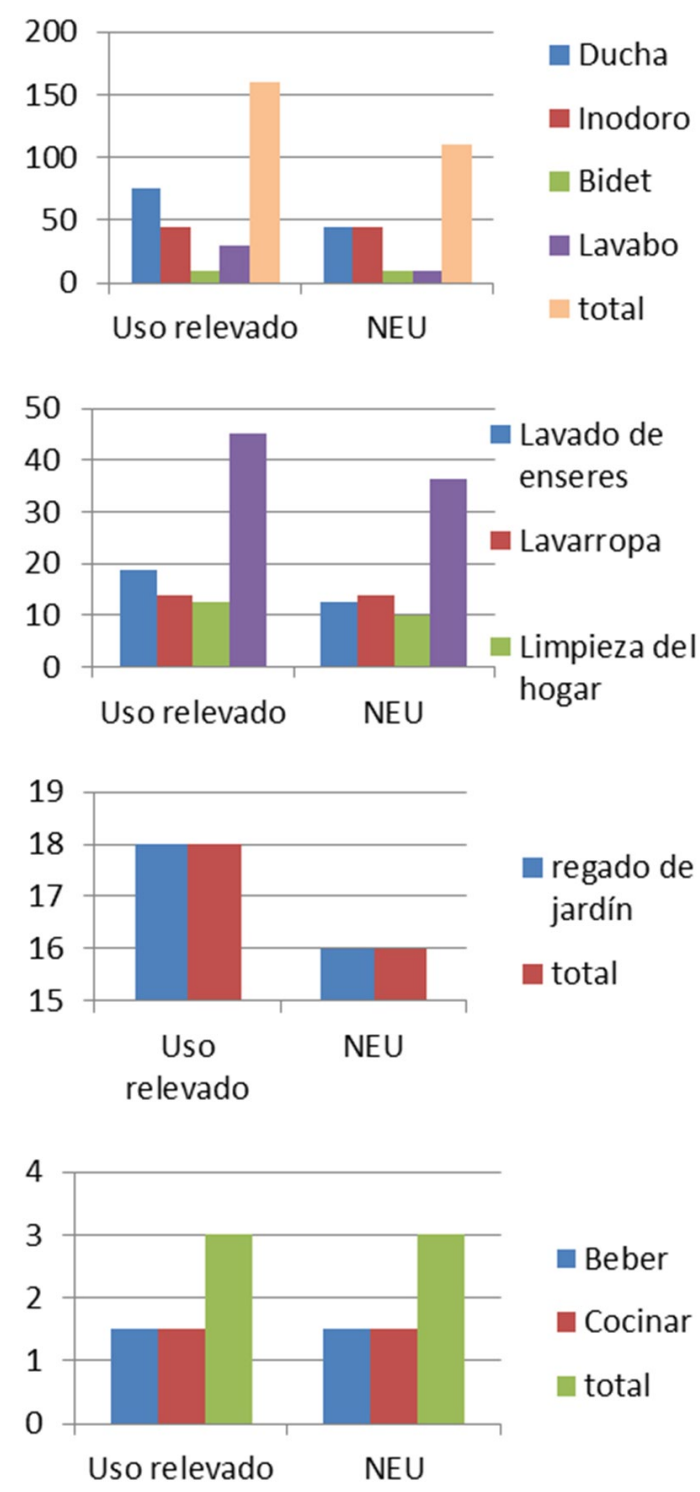

Figuras 9-10-11-12 (de izquierda a derecha y de arriba hacia abajo) Niveles de eficiencia posibles frente a los distintos usos. Fuente: Elaboración de los autores en base a datos de ASSA, AySA y Cátedra de Instalaciones FADU-UNL (2018), 


\begin{tabular}{|c|c|c|c|c|c|c|c|c|} 
usos & Higiene Personal & $\begin{array}{c}\text { Higiene } \\
\text { Hogar }\end{array}$ & $\begin{array}{c}\text { Mantenim. } \\
\text { Verde }\end{array}$ & Ingesta & Hig. Pers. Ab. & $\begin{array}{c}\text { Hig, } \\
\text { Hogar } \\
\text { Ab. }\end{array}$ & $\begin{array}{c}\text { Mant. } \\
\text { Verde } \\
\text { Ab. }\end{array}$ & $\begin{array}{c}\text { Ingesta } \\
\text { Ab. }\end{array}$ \\
\hline litros & 160 & 45 & 18 & 3 & -50 & $-8,75$ & -2 & 3 \\
\hline & $\begin{array}{c}\text { consumos relevados } \\
\text { promedios }\end{array}$ & & & & $\begin{array}{c}\text { abatimiento por nivel } \\
\text { de eficiencia del usuario }\end{array}$ & & & \\
\hline
\end{tabular}

Tabla 2. Tabla con datos de consumos relevados (demanda) y valores posibles de reducción (abatimiento) en litros de agua aplicando criterio de eficiencia en los usuarios. Fuente: Elaboración de los autores.

Para elaborar una síntesis de cada escenario de eficiencia, se retoma el concepto de "abatimiento" propuesto por Clerc et al. (2013), referido a la curva que muestra el impacto producido ante medidas de reducción de consumos, emisiones y usos energéticos, el cual permite establecer indicadores entre las distintas decisiones de mitigación y ahorro, a fin de planificar los esfuerzos asociados a la misma.

Desde esta perspectiva, se entiende a los costos como beneficios percibidos, al reemplazarse una tecnología o uso de referencia por otro alternativo. En concreto, se puede elaborar una curva de abatimiento, en tanto representación gráfica de los costos abatidos de un menú de opciones, junto a sus porcentajes de reducción. En este gráfico, es posible observar las diversas selecciones ordenadas, como también la información relevante para evaluar y comparar los efectos de distintas medidas. Asimismo, las curvas encuentran su fuente principal de conocimiento en el abatimiento de emisiones de $\mathrm{CO}_{2}$ y GEl, pero, con propósitos ilustrativos, se utiliza el concepto para elaborar un gráfico representativo del posible abatimiento en litros de agua consumidos en los ítems considerados por sobre el NEU.

De los 226l/h/d, aplicando los criterios de NEU, se puede lograr una eficiencia de hasta el $26.88 \%$, esto es, unos $60,75 \mathrm{l} / \mathrm{h} / \mathrm{d}$ (Tabla 2 y Figura 13).

\section{$2^{\circ}$ ESCENARIO - CAMBIO EN LA TECNOLOGÍA SIN USO DEL AGUA DE LLUVIA COSECHADA}

La segunda opción, cambio en las tecnologías sin incorporar la cosecha de agua, se refiere a todas las posibilidades en las cuales se pueden utilizar artefactos e instalaciones que sean más eficientes, sin bajar los niveles de confort ni modificar las necesidades básicas de consumo. Un ejemplo de ello sería utilizar mochilas de inodoro selectivas ${ }^{14}$ o válvulas, en lugar de depósitos tradicionales, o bien, usar griferías con pulsador-temporizador o automáticas, en vez de canillas tradicionales (Figuras 14, 15, 16, 17 y 18).

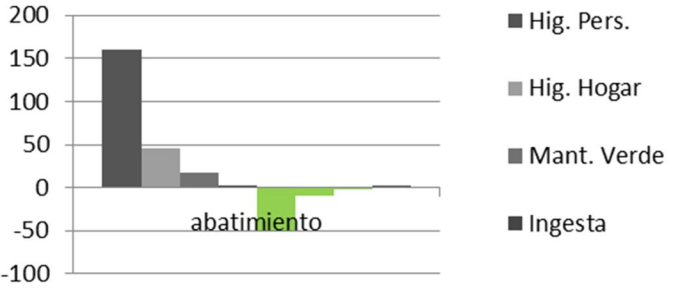

Figura 13: Gráfico de abatimiento en litros de agua aplicando criterio de eficiencia en los usuarios. Fuente: Elaboración de los autores.
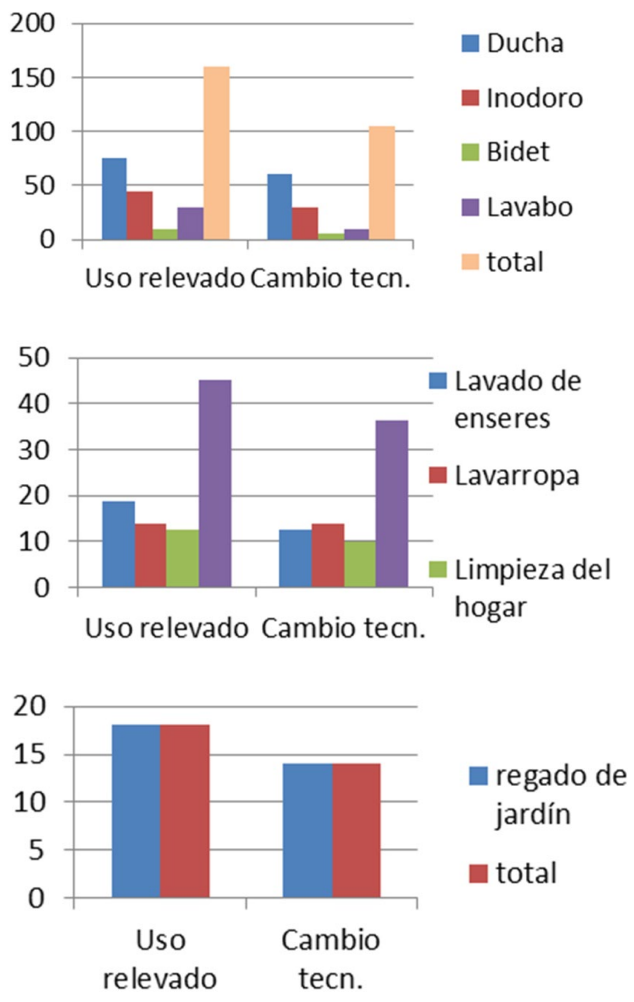

Figuras 14-15-16 (de izquierda a derecha y de arriba hacia abajo). Gráfico de barras donde se muestran los niveles de eficiencia posibles frente a los distintos usos. Fuente: Elaboración de los autores en base a datos de ASSA, AySA y Cátedra de Instalaciones FADU. 


\begin{tabular}{|c|c|c|c|c|c|c|c|c|}
\hline usos & Hig. Pers. & Hig. Hogar & $\begin{array}{c}\text { Mant. } \\
\text { Verde }\end{array}$ & Ingesta & $\begin{array}{c}\text { Hig. Pers. } \\
\text { Ab. }\end{array}$ & $\begin{array}{c}\text { Hig, Hogar } \\
\text { Ab. }\end{array}$ & $\begin{array}{c}\text { Mant. } \\
\text { Verde Ab. }\end{array}$ & Ingesta Ab. \\
\hline litros & 160 & 45 & 18 & 3 & -160 & $-26,25$ & -19 & 3 \\
\hline \multicolumn{3}{r}{ consumos relevados promedio } \\
\hline \multicolumn{3}{c|}{ abatimiento por consumo eficiente del usuario } \\
\hline
\end{tabular}

Tabla 3. Tabla con datos de consumos relevados (demanda) y valores posibles de reducción (abatimiento) en litros de agua, aplicando criterio de cambio en la tecnología sanitaria. Fuente: Elaboración de los autores.

\begin{tabular}{|c|c|c|c|c|}
\hline Casos & \multicolumn{3}{|c|}{ Posibilidad de uso del agua de lluvia cosechada } \\
& Higiene personal & Higiene hogar & Mantenim. verde & Consumo-ingesta \\
\hline Viv. P.B. & Disp. c/tecnología $*$ & Disponible & Disponible & No disponible \\
\hline Viv. Piso alto & Disponible & Disponible & Disponible & No disponible \\
\hline Viv. Edif. altura & No disponible & Disp. c/ limitac. ${ }^{*}$ & Disp. c/ limitac. ${ }^{*}$ & No disponible \\
\hline
\end{tabular}

Tabla 4. Casos y usos del agua de lluvia cosechada. Fuente: Elaboración de los autores.

${ }^{*}$ necesita de bombeo para incorporar el agua cosechada en la cisterna, hacia el tanque de reserva.

** solo posibilidad de uso en mantenimiento de verde, limpieza de veredas y áreas comunes (PB).

Solo a título ilustrativo, se presenta aquí una comparativa de algunos elementos ${ }^{15}$ :

- Válvula de descarga de inodoro primera marca: $\$ 7646$

- Mochila de inodoro de primera marca: $\$ 6550$

- Grifería de lavatorio, $4^{\text {to }}$ giro cierre cerámico de primera marca: $\$ 8345$

- Grifería de lavatorio, automática primera marca: $\$ 10320$.

De los $226 \mathrm{l} / \mathrm{h} / \mathrm{d}$, aplicando el criterio de eficiencia "cambios de las tecnologías", sin considerar el aprovechamiento del agua de lluvia cosechada, se puede lograr una eficiencia de hasta el $30 \%$, esto es, unos $67,80 \mathrm{l} / \mathrm{h} / \mathrm{d}$ (Tabla 3).

\section{$3^{\circ}$ ESCENARIO - CAMBIO EN LA TECNOLOGÍA CON USO DEL AGUA DE LLUVIA COSECHADA}

Incorporar en el uso sanitario la cosecha de agua disponible, aprovechando la obligatoriedad de implantación según la Ordenanza No 11959 de Santa Fe "Regulación de excedentes pluviales", presenta un amplio abanico de posibilidades, tanto en tipos de usos como en casos.

En cuanto al tipo edilicio: a diferencia de los dos escenarios de eficiencia presentados ut supra, que no implican cambios de implementación respecto al tipo de vivienda, vale decir, donde es posible aplicar un criterio de NEU y un cambio en la tecnología, tanto en viviendas de planta baja

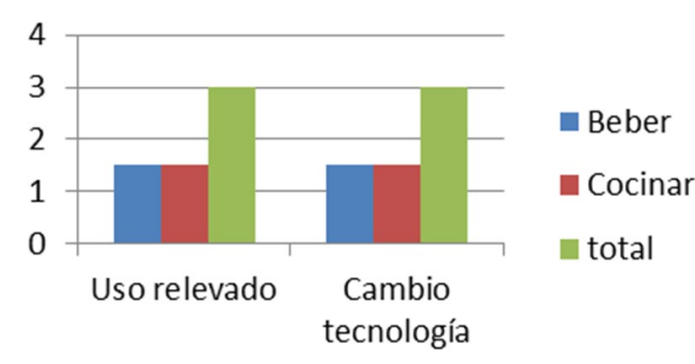

Figuras 17 Gráfico de barras donde se muestran los niveles de eficiencia posibles frente a los distintos usos. Fuente: Elaboración de los autores en base a datos de ASSA, AySA y Cátedra de Instalaciones FADU.

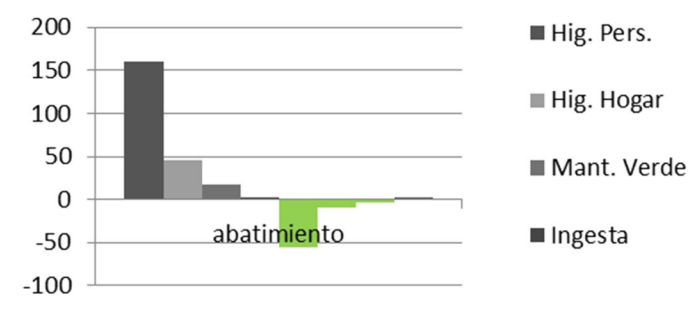

Figura 18. Gráfico de abatimiento en litros de agua aplicando criterio de cambio en la tecnología sanitaria. Fuente: Elaboración de los autores.

15 Referencia de una investigación que considera elementos disponibles en el mercado local de la ciudad de Santa Fe y en primeras marcas, como FV, Ferrum y Roca. Valores en pesos argentinos al mes de diciembre de 2019. Dólar de referencia \$ 62,5 (pesos argentinos). 


\begin{tabular}{|c|c|c|c|c|c|c|c|c|}
\hline usos & Hig. Pers. & Hig. Hogar & Mant. Verde & Ingesta & Hig. Pers. Ab. & $\begin{array}{l}\text { Hig, Hogar } \\
\text { Ab. }\end{array}$ & $\begin{array}{c}\text { Mant. Verde } \\
\text { Ab. }\end{array}$ & Ingesta $\mathrm{Ab}$. \\
\hline litros & 160 & 45 & 18 & 3 & -160 & $-26,25$ & -19 & 3 \\
\hline & $\begin{array}{l}\text { consumos } \\
\text { relevados } \\
\text { promedio }\end{array}$ & & & & $\begin{array}{c}\text { abatimiento } \\
\text { por consumo } \\
\text { eficiente del } \\
\text { usuario }\end{array}$ & & & \\
\hline
\end{tabular}

Tabla 5. Tabla con datos de consumos relevados (demanda) y valores posibles de reducción (abatimiento) en litros de agua aplicando criterio de utilización del agua de lluvia. Fuente: Elaboración de los autores.

como de pisos altos y en edificios de vivienda multifamiliar, en este tercer escenario, se presentan restricciones según el tipo edilicio: viviendas de una planta, viviendas de pisos altos y viviendas en edificios multifamiliares o en altura. Las primeras de ellas -considerando el nivel piezómetro disponible en la ciudad de Santa Fe- no necesitan tanque de bombeo pero sí de reserva, y pueden contar con agua de red directa en cocina, por ejemplo, para consumo e ingesta. En las segundas, viviendas desarrolladas en dos niveles, la planta baja y el primer piso, necesitan de tanque de bombeo y de reserva, pero con posibilidad de consumos en servicio directo. Las terceras, por su parte, requieren de tanque de bombeo y reserva, sin posibilidad de servicios directos.

En cuanto al uso: los usos, en relación con el aprovechamiento de la cosecha de agua de lluvia, se presentan caracterizados por dos grupos: el primero, utilización simple, esto es, solo utilización desde la cisterna; y, el segundo, uso complejo, o sea, incorporación del agua recogida en el circuito hidráulico sanitario, con la excepción del consumo e ingesta. Se considera a este último como significativo, ya que solo puede lograrse un valor de eficiencia en esa situación. Efectivamente, si se aprovecha el agua acumulada, sin incorporarla al circuito hidráulico, sólo con la extracción de la cisterna a nivel, mediante una canilla, su utilización queda limitada a higiene de pisos, veredas y mantenimiento de verde, todos consumos que no inciden directamente sobre el grueso del promedio diario.

Las distintas posibilidades en función de los tres casos de viviendas considerados, se pueden resumir en la siguiente tabla (Tabla 4)

En virtud de lo evidenciado, el agua meteórica acumulada no puede ser aprovechada de manera significativa en edificios en altura, por lo que su uso, en el marco de lo establecido por la reglamentación vigente -Ord. 11595-, queda restringido solamente a pequeñas acciones: higiene de espacios comunitarios, riego de verdes, entre otros. Por ende, limitaremos las consideraciones a viviendas, tanto en planta baja como de pisos altos (Tabla 5 y Figuras 19, 20, 21,22 y 23). Si contemplamos una situación hipotética e ideal, en donde no existiesen limitaciones en la cantidad de agua caída ni en la capacidad de almacenamiento, estas se podrían abatir, en el sentido de reemplazar el consumo
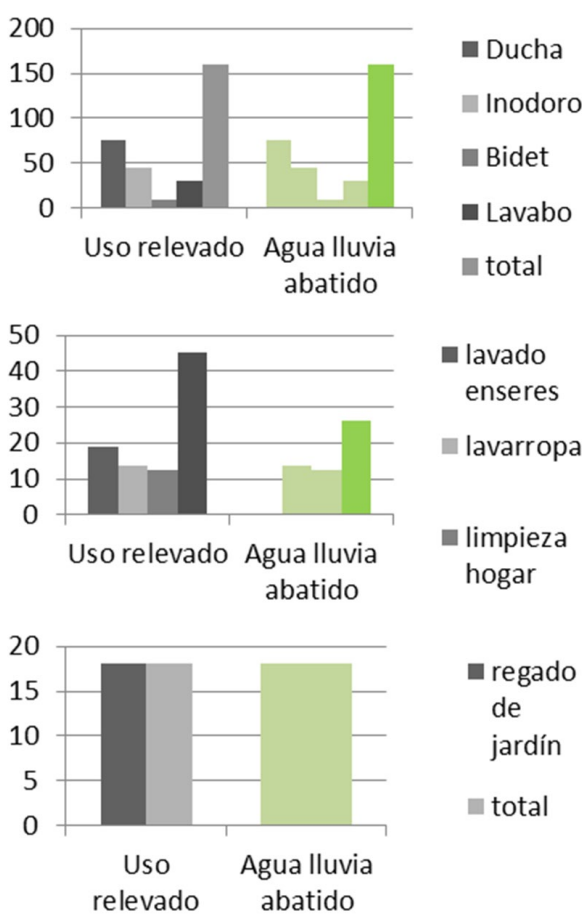

regado

de

jardín

total

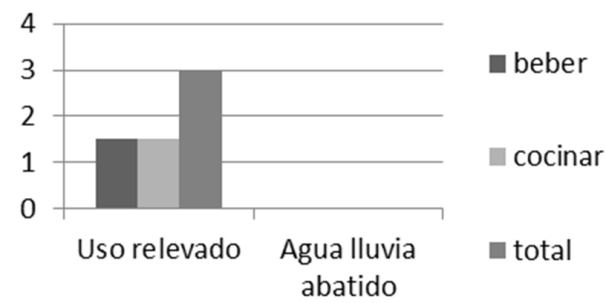

Figuras 19-20-21-22: Porcentajes abatidos en litros de agua por cada nivel de uso, higiene personal, higiene del hogar, mantenimiento de verde y consumo e ingesta. Fuente: Elaboración de los autores.

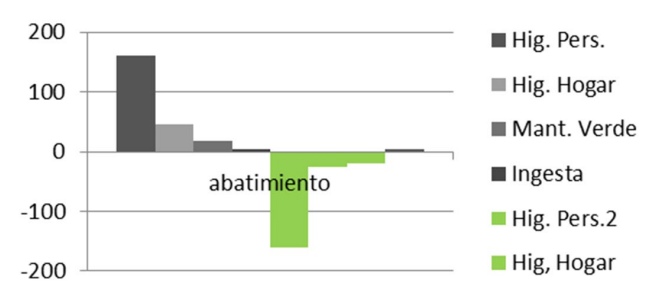

Figura 23. Gráfico resumen de abatimiento en litros de agua aplicando criterio de utilización del agua de lluvia. Fuente: Elaboración de los autores. 
Tabla 6. Tabla con datos de consumos mensuales (demanda) y valores posibles de reducción (abatimiento) en litros de agua, que considera el promedio anual de lluvias en Santa Fe y la capacidad de almacenamiento de las cisternas, según Ordenanza $\mathrm{N}^{\circ} 11595$ y para el caso de estudio ejemplificado. Fuente: Elaboración de los autores.

del servicio de red por agua de lluvia, del siguiente modo: En el gráfico de higiene personal, se puede abatir el 100\%, esto es, 160l/h/d de consumo de agua. En el gráfico de higiene del hogar, es posible abatir el 58\%, es decir, $26,25 \mathrm{l} / \mathrm{h} / \mathrm{d}$. El lavado de enseres se supone aquí en la pileta de cocina $y$, como se explicará, constituye un consumo surtido por agua de red. En el gráfico de mantenimiento de verde, se puede abatir el 100\%: 18l/h/d. Por último, en el gráfico de consumo de agua, no es posible abatir, dado que la ingesta para beber y cocinar se presupone por defecto en agua de red, la cual garantiza la aptitud técnica (Figura 24). Esta situación ideal, en la ciudad, posee una limitación tanto en cantidad de agua caída como en la reserva. Para reemplazar este escenario ideal por uno real, se propone remitir al ejemplo mencionado anteriormente, en donde "una vivienda con una cubierta de $100 \mathrm{~m}^{2}$ que recibe $1000 \mathrm{~mm}$ a $1200 \mathrm{~mm}$ de agua anual, recogerá de 120000 Its a 144000 Its por año. La cantidad resultante arroja un promedio mensual de 10000 a 12000 litros". Si son necesarios $226 \mathrm{l} / \mathrm{h} / \mathrm{d}$, para un hogar promedio se requieren mensualmente 27120 litros de agua, vale decir que, simplificando a una función lineal de abatimiento y por cada ítem, se podría conseguir un aprovechamiento del recurso del $44 \%$. Si a esto se suma que la Ord. $\mathrm{N}^{\circ}$ 11595 establece volúmenes de diseño que, para este ejemplo de vivienda tipo con cuatro integrantes y $100 \mathrm{~m}^{2}$ de superficies impermeables, alcanzan los 6000 litros de cosecha mensuales, el abatimiento posible sería del orden del 22\% (Tabla 6).

\section{RESULTADOS Y DISCUSIÓN}

Sobre los resultados del estudio, y en el marco de los tres escenarios abordados por el análisis, se plantean las siguientes consideraciones.

En el primer escenario, con un adecuado Nivel de Eficiencia del Usuario (NEU), se logran valores de eficiencia de hasta el $27 \%$, los cuales señalan claramente la importancia de los habitantes en relación al uso y la necesidad de informar, capacitar y difundir las virtudes del uso racional del recurso. En el segundo escenario, utilizar tecnologías disponibles en el mercado local con criterios de eficiencia y sin mayores costos, permiten un abatimiento de hasta el 30\%, lo cual indica, entre otros, la relevancia del criterio, responsabilidad y conocimiento de los profesionales al realizar proyectos y ejecuciones de obras. El tercer escenario posibilita abatimientos de entre el $22 \%$ al $44 \%$, lo que implica un

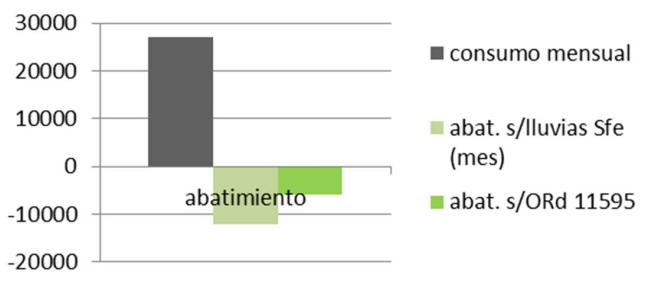

Figura 24. Gráfico de abatimiento en litros de agua, que aplica criterio de uso de agua de lluvia considerando promedio anual y capacidad de almacenamiento para el ejemplo explicitado. Fuente: Elaboración de los autores.

compromiso del profesional y del usuario, no solamente con el cumplimiento de una ordenanza, sino para con el medio y los recursos. De lo expuesto, se desprende que la cosecha de agua de lluvia, apta para diversos usos, puede representar un significativo aporte respecto al agua consumida de la red, llegando a valores de orden de reducción de cerca del 40\%.

Por otro lado, a través de la descripción del sistema hídrico de la ciudad, o sea, el modo en que se consume el agua de red y las disposiciones legales vigentes en materia de regulación de excedentes pluviales, se puede inferir que el sistema de acumulación de agua de lluvia es eficaz, en el sentido de que cumple con un propósito. Sin embargo, en los términos en que se pretende lograr lo indispensable con el aprovechamiento de lo disponible, no resulta eficiente en cuanto al uso de recursos.

\section{CONCLUSIONES}

Como ha quedado expuesto, si bien la decisión de acumular los excedentes y su eliminación paulatina posterior atiende al cumplimiento de la legislación vigente, no promueve márgenes de eficiencia: aun cuando los usos se superponen, el agua cosechada no contribuye a disminuir el consumo de provisión de red.

Paralelamente, la normativa reciente establece valores asociados con los aspectos morfológicos de las edificaciones, sin embargo, se presentan sólo a efectos de orientar los cálculos correspondientes: pendientes, superficies de escurrimiento, tamaño de los desagües, etc. En efecto, aún no se registran expresos lineamientos que, en términos de diseño, permitan optimizar la reutilización del recurso agua, sin detrimento de su calidad de servicio y para satisfacer los mínimos de demanda analizados. 
Por último, cabe señalar que el resultado del estudio, sin discutir la normativa en el marco del sistema urbano, pretende aportar a la reflexión sobre aquellas decisiones de la praxis proyectual que, en el marco de la construcción de infraestructuras para optimizar el consumo del recurso agua en Santa $\mathrm{Fe}$, no promueven la eficiencia como valor agregado y, por lo tanto, desalientan la concepción de la vivienda como hábitat sustentable.

\section{REFERENCIAS BIBLIOGRÁFICAS}

Alonso-Frank, A., Kuchen, E. (2017). Validación de la herramienta metodológica de Alonso-Frank \& Kuchen para determinar el indicador de nivel de eficiencia energética del usuario de un edificio residencial en altura, en San Juan, Argentina. Revista Hábitat Sustentable. Volumen 7 (1), pp. 6-13.

Bartram, J., Corrales, L., Davison, A., Deere, D., Drury, D., Gordon, B., Howard, G., y Stevens, M. (2009). Manual para el desarrollo de planes de seguridad del agua: metodología pormenorizada de gestión de riesgos para proveedores de agua de consumo. Ginebra: Organización Mundial de la Salud.

Bello, O., Ballesteros, J., Buitrago, M., González, M. y Velasco, O. (2018). Análisis retrospectivo de las inundaciones: lecciones y recomendaciones. Buenos Aires: CEPAL - ONU.

Calcagno, A., Mendiburo, N., Gaviño Novillo, J.M. (2000). Informe sobre la gestión del agua en Argentina. Buenos Aires: Ed. Global Water Partnership.

Castillo-Ávalos, Y. y Rovira-Pinto, A. (2013). Eficiencia hídrica en la vivienda. Revista Tecnología y ciencias del agua. Volumen 4 (4), pp.159-171.

Cátedra de Instalaciones I, II y III (2018). Cuadernillo bibliográfico de Instalaciones [Apuntes]. Taller Vertical Bellot. FADU. UNL.

Clerc, J., Díaz, M. y Campos, B. (2013). Desarrollo de una metodología para la construcción de curvas de abatimiento de emisiones de GEl incorporando la incertidumbre asociada a las principales variables de mitigación. Banco Interamericano de Desarrollo.

Delucchi, A. (2016). Acerca del proyecto en arquitectura. Entre la reconstrucción y la invención. Buenos Aires: Ed. Diseño.

Díaz Dorado, M.D. (2008). Instalaciones sanitarias y contra incendios en edificios. Buenos Aires: Ed. Alsina.

Franco, F. (2019). Cuadernillo del Laboratorio de Hidráulica. [Apuntes] Facultad de Ingeniería en Ciencias Hídricas (FICH). Universidad Nacional del Litoral.

Jiménez, A. (1994). Capítulo 1. En H. Garduño y F. Arreguín (Eds.), Uso eficiente del agua (pp. 6-26). Montevideo: UNESCO-ORCYT.

Latour, B. (2017). Cara a cara con el planeta. Una nueva mirada sobre el cambio climático alejada de las posiciones apocalípticas. Buenos Aires: Siglo Veintiuno Editores.

Lemme, J. C. (1973). Instalaciones aplicadas en los edificios. Buenos Aires: Luis y Félix Fossati SRL.
Paoli, C. y Schreider, M. (Eds.). (2000). El Río Paraná en su tramo medio. (Vol. 1). Santa Fe: Centro de Publicaciones UNL.

Sánchez, L.D. y Sánchez A. (2004). Uso eficiente del agua. Ponencias sobre una perspectiva general temática (TOP). Instituto de Investigación y Desarrollo en Agua Potable, Saneamiento Básico y Conservación del Recurso Hídrico (CINARA). Internationa Water and Sanitation Centre (IRC). 